\title{
Quantum mechanics gives stability to a Nash equilibrium.
}

\author{
A. Iqbal and A.H. Toor \\ Electronics Department, Quaid-i-Azam University, \\ Islamabad, Pakistan. \\ email: qubit@isb.paknet.com.pk
}

October 25, 2018

\begin{abstract}
We consider a slightly modified version of the Rock-Scissors-Paper (RSP) game from the point of view of evolutionary stability. In its classical version the game has a mixed Nash equilibrium (NE) not stable against mutants. We find a quantized version of the RSP game for which the classical mixed NE becomes stable.
\end{abstract}

\section{Introduction}

Long played as a children's pastime, or as an odd-man-out selection process, the Rock-Scissors-Paper (RSP) is a game for two players typically played using the players' hands. The two players opposite each others, tap their fist in their open palm three times (saying Rock, Scissors, Paper) and then show one of three possible gestures. The Rock wins against the scissors (crushes it) but looses against the paper (is wrapped into it). The Scissors wins against the paper (cuts it) but looses against the rock (is crushed by it). The Paper wins against the rock (wraps it) but looses against the scissors (is cut by it).

In a slightly modified version of the RSP game both players get a small premium $\epsilon$ for a draw. This game can be represented by the following payoff matrix

$$
\left(\begin{array}{cccc} 
& R & S & P \\
R & -\epsilon & 1 & -1 \\
S & -1 & -\epsilon & 1 \\
P & 1 & -1 & -\epsilon
\end{array}\right)
$$

where $-1<\epsilon<0$. The matrix of the usual game is obtained when $\epsilon$ is zero in the matrix (11). 
One cannot win if one's opponent knew which strategy was going to be picked. For example, picking Rock consistently all the opponent needs to do is pick Paper and s/he would win. Players find soon that in case predicting opponent's strategy is not possible the best strategy is to pick Rock, Scissors, or Paper at random. In other words, the player selects Rock, Scissors, or Paper with a probability of $\frac{1}{3}$. In case opponent's strategy is predictable picking a strategy at random with a probability of $\frac{1}{3}$ is not the best thing to do unless the opponent is doing the same [2].

We explore Evolutionarily Stable Strategies (ESSs) in quantized RSP game. Originally defined by Smith and Price [6] as a behavioral phenotype an ESS cannot be invaded by a mutant strategy when a population is playing it. A mutant strategy does things in different ways than most of a population does. Smith and Price considered a symmetric game where the players are anonymous. Let $P[u, v]$ be the payoff to a player playing $u$ against the player playing $v$. Strategy $u$ is an ESS if for any alternative strategy $v$, the following two requirements are satisfied

$$
P[u, u] \geq P[v, u]
$$

and in the case $P[u, u]=P[v, u]$

$$
P[u, v]>P[v, v]
$$

Requirement (2) is in fact the Nash requirement and says that no single individual can gain by unilaterally changing her strategy from $u$ to $v$. An ESS is in fact a stable NE in a symmetric game and its stability is against a small group of mutants [6, 7].

A straight analysis of the modified RSP game of matrix (11) shows that playing each of the three different pure strategies with a fixed equilibrial probability $\frac{1}{3}$ constitutes a mixed NE. However it is not an ESS because $\epsilon$ is negative [7].

In an earlier paper [3] we showed that in quantized version of certain asymmetric games between two players it is possible to make appear or disappear an ESS that is a pure strategy pair NE by controlling the initial state used to play the game. Because a classical game is embedded in its quantized form, therefore, it is possible that a pure strategy pair NE remains intact in both classical and certain quantized form of the same game but is an ESS in only one form. Later we presented an example [4] of a symmetric game between two players for which a pure strategy NE is an ESS in classical version of the game but not so in a quantized form even when it remains a NE in both versions. This is more relevant because the idea of an ESS was originally defined for symmetric contests. We also showed [5] that mixed strategy ESSs can be related to entanglement and can be affected by quantization for three player games. However it is not the case for two player games when the quantum state is in a simpler form proposed by Marinatto and Weber $(\mathrm{M} \& W)$ [1] in their scheme to quantize a two player game in normal form. 
M\&W [1] expanded on the scheme proposed by Eisert et al [15] for the game of Prisoner's Dilemma. Eisert et al showed that dilemma doesn't exist in a quantum version of the game. Their motivation of $\mathrm{M} \& \mathrm{~W}$ was to remove the need of an unentangling gate in Eisert's scheme [8, 9]. In our effort to extend the ideas of evolutionary game theory toward quantum games we found M\&W's scheme more suitable for following reasons:

(a). In usual setup of a symmetric bi-matrix evolutionary game two pure strategies are assumed such that players can play a mixed strategy by their probabilistic combination. In a similar way players in M\&W's scheme can play a mixed strategy by applying two unitary operators in their possession with classical probabilities.

(b): The usual definition of 'fitness' of a mixed strategy in evolutionary games [2] can be given a straightforward extension in M\&W's scheme [3]. It is done when in the quantum game players use only one unitary operator out of the two.

(c): The theory of ESSs in evolutionary game theory is developed mostly for situations when players are anonymous and possess a discrete number of pure strategies. We find that ESS idea can be extended towards quantum settings more easily in M\&W's scheme than in Eisert's scheme involving a continuum of pure strategies players have option to play. The idea of an ESS as a stable equilibrium is confronted with problems when players have an access to a continuum of pure strategies 10].

In this paper we want to extend our previous results regarding effects of quantization on evolutionary stability for a modified version of the RSP game. This game is different because now classically each player possesses three pure strategies instead of two. A classical mixed NE exists that is not an ESS. Our motivation is to explore the possibility that the classical mixed NE becomes an ESS for some initial entangled quantum state. We show that such a quantum state not only exists but also easy to find.

\section{Quantized RSP game}

Using simpler notation: $R \sim 1, S \sim 2, P \sim 3$ we quantize this game via M\&W's scheme [1]. We allow the two players are in possession of three unitary operators $I, C$ and $D$ defined as

$$
\begin{array}{lll}
I|1\rangle=|1\rangle & C|1\rangle=|3\rangle & D|1\rangle=|2\rangle \\
I|2\rangle=|2\rangle & C|2\rangle=|2\rangle & D|2\rangle=|1\rangle \\
I|3\rangle=|3\rangle & C|3\rangle=|1\rangle & D|3\rangle=|3\rangle
\end{array}
$$

where $C^{\dagger}=C=C^{-1}$ and $D^{\dagger}=D=D^{-1}$ and $I$ is identity operator. We also start from a general payoff matrix for two players each having three strategies 


$$
\left[\begin{array}{cccc} 
& 1 & 2 & 3 \\
1 & \left(\alpha_{11}, \beta_{11}\right) & \left(\alpha_{12}, \beta_{12}\right) & \left(\alpha_{13}, \beta_{13}\right) \\
2 & \left(\alpha_{21}, \beta_{21}\right) & \left(\alpha_{22}, \beta_{22}\right) & \left(\alpha_{23}, \beta_{23}\right) \\
3 & \left(\alpha_{31}, \beta_{31}\right) & \left(\alpha_{32}, \beta_{32}\right) & \left(\alpha_{33}, \beta_{33}\right)
\end{array}\right]
$$

where $\alpha_{i j}, \beta_{i j}$ are payoffs to Alice and Bob respectively when Alice plays $i$ and Bob plays $j$ and $1 \leq i, j \leq 3$. Suppose Alice and Bob apply operators $C D$ and $I$ with probabilities $p, p_{1}$ and $\left(1-p-p_{1}\right)$ and $q, q_{1}$ and $\left(1-q-q_{1}\right)$ respectively. Let represent the initial state of the game by $\rho_{i n}$. After Alice plays her strategy the state changes to

$$
\stackrel{A}{\rho_{\text {in }}}=\left(1-p-p_{1}\right) I_{A} \rho_{i n} I_{A}^{\dagger}+p C_{A} \rho_{i n} C_{A}^{\dagger}+p_{1} D_{A} \rho_{i n} D_{A}^{\dagger}
$$

The final density matrix after Bob too has played his strategy is

$$
\stackrel{A_{\rho}, B}{\rho}=\left(1-q-q_{1}\right) I_{B}{\stackrel{A}{\rho_{i n}}}_{I_{B}^{\dagger}}^{\dagger}+q C_{B} \rho_{i n}^{A} C_{B}^{\dagger}+q_{1} D_{B}{ }_{i n}^{A} D_{B}^{\dagger}
$$

This density matrix can be written as

$$
\begin{aligned}
\stackrel{A_{\rho}, B}{\rho_{f}}= & \left(1-p-p_{1}\right)\left(1-q-q_{1}\right)\left\{I_{A} \otimes I_{B} \rho_{i n} I_{A}^{\dagger} \otimes I_{B}^{\dagger}\right\}+p\left(1-q-q_{1}\right) \times \\
& \left\{C_{A} \otimes I_{B} \rho_{i n} C_{A}^{\dagger} \otimes I_{B}^{\dagger}\right\}+p_{1}\left(1-q-q_{1}\right)\left\{D_{A} \otimes I_{B} \rho_{i n} D_{A}^{\dagger} \otimes I_{B}^{\dagger}\right\}+ \\
& \left(1-p-p_{1}\right) q\left\{I_{A} \otimes C_{B} \rho_{i n} I_{A}^{\dagger} \otimes C_{B}^{\dagger}\right\}+p q\left\{C_{A} \otimes C_{B} \rho_{i n} C_{A}^{\dagger} \otimes C_{B}^{\dagger}\right\}+ \\
& p_{1} q\left\{D_{A} \otimes C_{B} \rho_{i n} D_{A}^{\dagger} \otimes C_{B}^{\dagger}\right\}+\left(1-p-p_{1}\right) q_{1}\left\{I_{A} \otimes D_{B} \rho_{i n} I_{A}^{\dagger} \otimes D_{B}^{\dagger}\right\} \\
& +p q_{1}\left\{C_{A} \otimes D_{B} \rho_{i n} C_{A}^{\dagger} \otimes D_{B}^{\dagger}\right\}+p_{1} q_{1}\left\{D_{A} \otimes D_{B} \rho_{i n} D_{A}^{\dagger} \otimes D_{B}^{\dagger}\right\}
\end{aligned}
$$

The basis vectors of initial quantum state with three pure classical strategies are $|11\rangle,|12\rangle,|13\rangle,|21\rangle,|22\rangle,|23\rangle,|31\rangle,|32\rangle$, and $|33\rangle$. Setting the initial quantum state to following general form

$$
\begin{aligned}
\left|\psi_{\text {in }}\right\rangle= & c_{11}|11\rangle+c_{12}|12\rangle+c_{13}|13\rangle+c_{21}|21\rangle+c_{22}|22\rangle+c_{23}|23\rangle+ \\
& c_{31}|31\rangle+c_{32}|32\rangle+c_{33}|33\rangle
\end{aligned}
$$

with normalization

$$
\begin{aligned}
& \left|c_{11}\right|^{2}+\left|c_{12}\right|^{2}+\left|c_{13}\right|^{2}+\left|c_{21}\right|^{2}+\left|c_{22}\right|^{2}+\left|c_{23}\right|^{2}+ \\
& \left|c_{31}\right|^{2}+\left|c_{32}\right|^{2}+\left|c_{33}\right|^{2}=1
\end{aligned}
$$

and writing payoff operators for Alice and Bob as [1] 


$$
\begin{aligned}
\left(P_{A, B}\right)_{\text {oper }}= & (\alpha, \beta)_{11}|11\rangle\left\langle 11\left|+(\alpha, \beta)_{12}\right| 12\right\rangle\left\langle 12\left|+(\alpha, \beta)_{13}\right| 13\right\rangle\langle 13|+ \\
& (\alpha, \beta)_{21}|21\rangle\left\langle 21\left|+(\alpha, \beta)_{22}\right| 22\right\rangle\left\langle 22\left|+(\alpha, \beta)_{23}\right| 23\right\rangle\langle 23|+ \\
& (\alpha, \beta)_{31}|31\rangle\left\langle 31\left|+(\alpha, \beta)_{32}\right| 32\right\rangle\left\langle 32\left|+(\alpha, \beta)_{33}\right| 33\right\rangle\langle 33|
\end{aligned}
$$

the payoffs to Alice or Bob can be obtained by taking a trace of $\left(P_{A, B}\right)_{\text {oper }} \stackrel{A, B}{\rho_{f}}$ i.e. [1]

$$
P_{A, B}=\operatorname{tr}\left[\left\{\left(P_{A, B}\right)_{o p e r}\right\} \stackrel{A, B}{\rho}{ }_{f}\right]
$$

Payoff to Alice, for example, can be written as

$$
P_{A}=\Phi \Omega \Upsilon^{T}
$$

where $T$ is for transpose and the matrices $\Phi, \Omega$, and $\Upsilon$ are

$$
\begin{aligned}
& \Phi=\left[\begin{array}{lll}
\left(1-p-p_{1}\right)\left(1-q-q_{1}\right) & p\left(1-q-q_{1}\right) & p_{1}\left(1-q-q_{1}\right)
\end{array}\right. \\
& \left.\left(1-p-p_{1}\right) q \quad p q \quad p_{1} q \quad\left(1-p-p_{1}\right) q_{1} \quad p q_{1} \quad p_{1} q_{1}\right] \\
& \Upsilon=\left[\begin{array}{lllllllll}
\alpha_{11} & \alpha_{12} & \alpha_{13} & \alpha_{21} & \alpha_{22} & \alpha_{23} & \alpha_{31} & \alpha_{32} & \alpha_{33}
\end{array}\right] \\
& \Omega=\left[\begin{array}{ccccccccc}
\left|c_{11}\right|^{2} & \left|c_{12}\right|^{2} & \left|c_{13}\right|^{2} & \left|c_{21}\right|^{2} & \left|c_{22}\right|^{2} & \left|c_{23}\right|^{2} & \left|c_{31}\right|^{2} & \left|c_{32}\right|^{2} & \left|c_{33}\right|^{2} \\
\left|c_{31}\right|^{2} & \left|c_{32}\right|^{2} & \left|c_{33}\right|^{2} & \left|c_{21}\right|^{2} & \left|c_{22}\right|^{2} & \left|c_{23}\right|^{2} & \left|c_{11}\right|^{2} & \left|c_{12}\right|^{2} & \left|c_{13}\right|^{2} \\
\left|c_{21}\right|^{2} & \left|c_{22}\right|^{2} & \left|c_{23}\right|^{2} & \left|c_{11}\right|^{2} & \left|c_{12}\right|^{2} & \left|c_{13}\right|^{2} & \left|c_{31}\right|^{2} & \left|c_{32}\right|^{2} & \left|c_{33}\right|^{2} \\
\left|c_{13}\right|^{2} & \left|c_{12}\right|^{2} & \left|c_{11}\right|^{2} & \left|c_{23}\right|^{2} & \left|c_{22}\right|^{2} & \left|c_{21}\right|^{2} & \left|c_{33}\right|^{2} & \left|c_{32}\right|^{2} & \left|c_{31}\right|^{2} \\
\left|c_{33}\right|^{2} & \left|c_{32}\right|^{2} & \left|c_{31}\right|^{2} & \left|c_{23}\right|^{2} & \left|c_{22}\right|^{2} & \left|c_{21}\right|^{2} & \left|c_{13}\right|^{2} & \left|c_{12}\right|^{2} & \left|c_{11}\right|^{2} \\
\left|c_{23}\right|^{2} & \left|c_{22}\right|^{2} & \left|c_{21}\right|^{2} & \left|c_{13}\right|^{2} & \left|c_{12}\right|^{2} & \left|c_{11}\right|^{2} & \left|c_{33}\right|^{2} & \left|c_{32}\right|^{2} & \left|c_{31}\right|^{2} \\
\left|c_{12}\right|^{2} & \left|c_{11}\right|^{2} & \left|c_{13}\right|^{2} & \left|c_{22}\right|^{2} & \left|c_{21}\right|^{2} & \left|c_{23}\right|^{2} & \left|c_{32}\right|^{2} & \left|c_{31}\right|^{2} & \left|c_{33}\right|^{2} \\
\left|c_{32}\right|^{2} & \left|c_{31}\right|^{2} & \left|c_{33}\right|^{2} & \left|c_{22}\right|^{2} & \left|c_{21}\right|^{2} & \left|c_{23}\right|^{2} & \left|c_{12}\right|^{2} & \left|c_{11}\right|^{2} & \left|c_{13}\right|^{2} \\
\left|c_{22}\right|^{2} & \left|c_{21}\right|^{2} & \left|c_{23}\right|^{2} & \left|c_{12}\right|^{2} & \left|c_{11}\right|^{2} & \left|c_{13}\right|^{2} & \left|c_{32}\right|^{2} & \left|c_{31}\right|^{2} & \left|c_{33}\right|^{2}
\end{array}\right]
\end{aligned}
$$

This payoff is for the general matrix given in eq. (何). In case an exchange of strategies by Alice and Bob also exchanges their respective payoffs the game is said to be symmetric. The idea of evolutionary stability in mathematical biology is generally considered in symmetric contests. In a symmetric contest payoff to a player is then defined by his strategy and not by his identity. Payoffs in classical mixed strategy game can be obtained from eq. (11) when initial state is $\left|\psi_{i n}\right\rangle=|11\rangle$ and the game is symmetric when $\alpha_{i j}=\beta_{j i}$ in the matrix (11). Similarly the quantum game played using the general quantum state of eq. (8) becomes symmetric when $\left|c_{i j}\right|^{2}=\left|c_{j i}\right|^{2}$ for all constants $c_{i j}$ in the initial quantum state of eq. (8). This condition should hold along with the requirement 
$\alpha_{i j}=\beta_{j i}$ on the matrix (4). The payoff to Alice or Bob i.e. $P_{A}, P_{B}$ then does not need a subscript and we can use only $P$.

We now come to the question of evolutionary stability in this quantized version of the RSP game.

\section{Evolutionary stability in the quantized RSP game}

We define a strategy by a pair of numbers $\left(p, p_{1}\right)$ when players are playing quantized RSP game. It is understood that the identity operator is ,then, applied with probability $1-p-p_{1}$. Similar to requirements in eq. (2) and eq. (3) the conditions making a strategy $\left(p^{\star}, p_{1}^{\star}\right)$ an ESS can now be written as [6]

$$
\begin{aligned}
\text { 1. } P\left\{\left(p^{\star}, p_{1}^{\star}\right),\left(p^{\star}, p_{1}^{\star}\right)\right\} & >P\left\{\left(p, p_{1}\right),\left(p^{\star}, p_{1}^{\star}\right)\right\} \\
\text { 2. if } P\left\{\left(p^{\star}, p_{1}^{\star}\right),\left(p^{\star}, p_{1}^{\star}\right)\right\} & =P\left\{\left(p, p_{1}\right),\left(p^{\star}, p_{1}^{\star}\right)\right\} \text { then } \\
P\left\{\left(p^{\star}, p_{1}^{\star}\right),\left(p, p_{1}\right)\right\} & >P\left\{\left(p, p_{1}\right),\left(p, p_{1}\right)\right\}
\end{aligned}
$$

Suppose $\left(p^{\star}, p_{1}^{\star}\right)$ is a mixed NE then

$$
\left\{\left.\frac{\partial P}{\partial p}\right|_{\substack{p=q=p^{\star} \\ p_{1}=q_{1}=p_{1}^{\star}}}\left(p^{\star}-p\right)+\left.\frac{\partial P}{\partial p_{1}}\right|_{\substack{p=q=p^{\star} \\ p_{1}=q_{1}=p_{1}^{\star}}}\left(p_{1}^{\star}-p_{1}\right)\right\} \geq 0
$$

Using substitutions

$$
\begin{aligned}
& \left|c_{11}\right|^{2}-\left|c_{31}\right|^{2}=\triangle_{1}, \quad\left|c_{21}\right|^{2}-\left|c_{11}\right|^{2}=\triangle_{1}^{\prime} \\
& \left|c_{13}\right|^{2}-\left|c_{33}\right|^{2}=\triangle_{2}, \quad\left|c_{22}\right|^{2}-\left|c_{12}\right|^{2}=\triangle_{2}^{\prime} \\
& \left|c_{12}\right|^{2}-\left|c_{32}\right|^{2}=\triangle_{3}, \quad\left|c_{23}\right|^{2}-\left|c_{13}\right|^{2}=\triangle_{3}^{\prime}
\end{aligned}
$$

we get

$$
\begin{array}{c|c}
\frac{\partial P}{\partial p} \quad \mid \begin{array}{c}
p=q=p^{\star} \\
p_{1}=q_{1}=p_{1}^{\star} \\
\end{array} p_{1}^{\star}\left(\triangle_{1}-\triangle_{3}\right)\left\{\left(\alpha_{11}+\alpha_{32}\right)-\left(\alpha_{12}+\alpha_{31}\right)\right\}- \\
& \triangle_{1}\left(\alpha_{11}-\alpha_{31}\right)-\triangle_{2}\left(\alpha_{13}-\alpha_{33}\right)-\triangle_{3}\left(\alpha_{12}-\alpha_{32}\right) \\
\frac{\partial P}{\partial p_{1}} \quad \mid \begin{array}{c}
p=q=p^{\star} \\
p_{1}=q_{1}=p_{1}^{\star}
\end{array} & p^{\star}\left(\triangle_{3}^{\prime}-\triangle_{1}^{\prime}\right)\left\{\left(\alpha_{11}+\alpha_{23}\right)-\left(\alpha_{13}+\alpha_{21}\right)\right\}+ \\
& p_{1}^{\star}\left(\triangle_{2}^{\prime}-\triangle_{1}^{\prime}\right)\left\{\left(\alpha_{11}+\alpha_{22}\right)-\left(\alpha_{12}+\alpha_{21}\right)\right\}+ \\
& \triangle_{1}^{\prime}\left(\alpha_{11}-\alpha_{21}\right)+\triangle_{2}^{\prime}\left(\alpha_{12}-\alpha_{22}\right)+\triangle_{3}^{\prime}\left(\alpha_{13}-\alpha_{23}\right)
\end{array}
$$

For the matrix (11) above equations can be written as 


$$
\begin{aligned}
& \frac{\partial P}{\partial p} \quad \mid \begin{array}{c}
p=q=p^{\star} \\
p_{1}=q_{1}=p_{1}^{\star}
\end{array}=\triangle_{1}\left\{-2 \epsilon p^{\star}-(3+\epsilon) p_{1}^{\star}+(1+\epsilon)\right\}+ \\
& \triangle_{2}\left\{2 \epsilon p^{\star}+(1-\epsilon)\right\}+\triangle_{3}\left\{(3+\epsilon) p_{1}^{\star}-2\right\} \\
& \frac{\partial P}{\partial p_{1}} \quad \begin{array}{c}
p=q=p^{\star} \\
p_{1}=q_{1}=p_{1}^{\star}
\end{array}=\triangle_{1}^{\prime}\left\{-p^{\star}(3-\epsilon)+2 \epsilon p_{1}^{\star}+(1-\epsilon)\right\}- \\
& \triangle_{2}^{\prime}\left\{2 \epsilon p_{1}^{\star}-(1+\epsilon)\right\}+\triangle_{3}^{\prime}\left\{(3-\epsilon) p^{\star}-2\right\}
\end{aligned}
$$

Also the payoff difference in the second condition of an ESS and given in eq. (14) reduces to

$$
\begin{aligned}
& P\left\{\left(p^{\star}, p_{1}^{\star}\right),\left(p, p_{1}\right)\right\}-P\left\{\left(p, p_{1}\right),\left(p, p_{1}\right)\right\} \\
= & \left(p^{\star}-p\right)\left[-\triangle_{1}\left\{2 \epsilon p+(3+\epsilon) p_{1}-(1+\epsilon)\right\}+\right. \\
& \left.\triangle_{2}\{2 \epsilon p+(1-\epsilon)\}+\triangle_{3}\left\{(3+\epsilon) p_{1}-2\right\}\right]+ \\
& \left(p_{1}^{\star}-p_{1}\right)\left[-\triangle_{1}^{\prime}\left\{(3-\epsilon) p-2 \epsilon p_{1}-(1-\epsilon)\right\}-\right. \\
& \left.\triangle_{2}^{\prime}\left\{2 \epsilon p_{1}-(1+\epsilon)\right\}+\triangle_{3}^{\prime}\{(3-\epsilon) p-2\}\right]
\end{aligned}
$$

With the substitutions $p^{\star}-p=x$ and $p_{1}^{\star}-p_{1}=y$ above payoff difference is

$$
\begin{aligned}
& P\left\{\left(p^{\star}, p_{1}^{\star}\right),\left(p, p_{1}\right)\right\}-P\left\{\left(p, p_{1}\right),\left(p, p_{1}\right)\right\} \\
= & \triangle_{1} x\{2 \epsilon x+(3+\epsilon) y\}-\triangle_{2}\left(2 \epsilon x^{2}\right)-\triangle_{3} x y(3+\epsilon)- \\
& \triangle_{1}^{\prime} y\{2 \epsilon y-(3-\epsilon) x\}+\triangle_{2}^{\prime}\left(2 \epsilon y^{2}\right)-\triangle_{3}^{\prime} x y(3-\epsilon)
\end{aligned}
$$

provided

$$
\left.\frac{\partial P}{\partial p}\right|_{\substack{p=q=p^{\star} \\ p_{1}=q_{1}=p_{1}^{\star}}}=\left.0 \quad \frac{\partial P}{\partial p_{1}}\right|_{\substack{p=q=p^{\star} \\ p_{1}=q_{1}=p_{1}^{\star}}}=0
$$

The conditions in eq. (23) together define the mixed NE $\left(p^{\star}, p_{1}^{\star}\right)$. Consider now the modified RSP game in classical form obtained by setting $\left|c_{11}\right|^{2}=1$ and all the rest of the constants to zero. The eqs. (23) now become

$$
\begin{array}{r}
-2 \epsilon p^{\star}-(\epsilon+3) p_{1}^{\star}+(\epsilon+1)=0 \\
(-\epsilon+3) p^{\star}-2 \epsilon p_{1}^{\star}+(\epsilon-1)=0
\end{array}
$$

and $p^{\star}=p_{1}^{\star}=\frac{1}{3}$ is obtained as a mixed NE for all the range $-1<\epsilon<0$. From eq. 22 we get

$$
\begin{aligned}
& P\left\{\left(p^{\star}, p_{1}^{\star}\right),\left(p, p_{1}\right)\right\}-P\left\{\left(p, p_{1}\right),\left(p, p_{1}\right)\right\} \\
= & 2 \epsilon\left(x^{2}+y^{2}+x y\right)=\epsilon\left\{(x+y)^{2}+\left(x^{2}+y^{2}\right)\right\} \leq 0
\end{aligned}
$$


In the classical form of the RSP game, therefore, the mixed NE $p^{\star}=p_{1}^{\star}=\frac{1}{3}$ is a NE but not an ESS because the second condition of ESS given in eq. (14) does not hold.

Define now a new initial state as follows

$$
\left|\psi_{\text {in }}\right\rangle=\frac{1}{2}\{|12\rangle+|21\rangle+|13\rangle+|31\rangle\}
$$

and use it to play the game instead of the classical game obtained from $\left|\psi_{i n}\right\rangle=$ $|11\rangle$. The strategy $p^{\star}=p_{1}^{\star}=\frac{1}{3}$ still forms a mixed NE because the conditions given by eq. (23) hold true for it. However the payoff difference of eq. (22) is now given below when $-1<\epsilon<0$, and $x, y \neq 0$

$$
\begin{aligned}
& P\left\{\left(p^{\star}, p_{1}^{\star}\right),\left(p, p_{1}\right)\right\}-P\left\{\left(p, p_{1}\right),\left(p, p_{1}\right)\right\} \\
= & -\epsilon\left\{(x+y)^{2}+\left(x^{2}+y^{2}\right)\right\}>0
\end{aligned}
$$

Therefore, the mixed Nash equilibrium $p^{\star}=p_{1}^{\star}=\frac{1}{3}$ not existing as an ESS in the classical form of the RSP game becomes an ESS when the game is quantized and played using the initial entangled quantum state given by the eq. (26).

Note that from eq. (11) the payoff sum to Alice and Bob $P_{A}+P_{B}$ can be obtained for both classical mixed strategy game (i.e. $\left|\psi_{i n}\right\rangle=|12\rangle$ ) and the quantum game played using the quantum state of eq. (26). For the matrix (11) we write these sums as $\left(P_{A}+P_{B}\right)_{c l}$ and $\left(P_{A}+P_{B}\right)_{q u}$ for classical mixed strategy and quantum games respectively and find

$$
\left(P_{A}+P_{B}\right)_{c l}=-2 \epsilon\left\{\left(1-p-p_{1}\right)\left(1-q-q_{1}\right)+p_{1} q_{1}+p q\right\}
$$

and

$$
\left(P_{A}+P_{B}\right)_{q u}=-\left\{\frac{1}{2}\left(P_{A}+P_{B}\right)_{c l}+\epsilon\right\}
$$

In case $\epsilon=0$ both the classical and quantum games are clearly zero sum. For our slightly modified version of the RSP game we have $-1<\epsilon<0$ and both versions of the game become non zero sum.

\section{Discussion}

Game theoretical modelling of interactions between living organisms in natural word has been developed mostly during the last three decades. Use of matrix games is quite common in areas such as theoretical and mathematical biology. The RSP game that we investigate in present paper is also played in nature like many other games. Lizards in the Coast Range of California play this 
game using three alternative male strategies locked in an ecological never ending process from which there seems little escape. On the other hand the recently developed quantum game theory has been shown to find applications in quantum information 17]. Though there is no evidence yet the possibility of quantum games being played at molecular level was hinted by Dawkins [18]. Trying to find the relevance of ideas from population biology in quantum settings is something that we call an inspiration from Dawkins' ideas.

The possibility of quantum mechanics playing a more direct role in life than binding together atoms has attracted much attention [12, 11]. Quantum mechanics 'fast tracking' a chemical soup to states that are biological and complex is an idea about which physicists from many areas have expressed opinions and the debate still continues. Supersymmetry in particle physics giving a unified description of fermions and bosons have also been suggested to provide an explanation of coding assignments in genetic code [13]. Patel's idea of quantum dynamics having a role in the DNA replication is another interesting suggestion [14. Quantum game theory [15, 16] can also have possibly interesting contributions to make towards attempts to understand quantum mechanical role in life.

Mathematical biologists have successfully developed mathematical models of evolution, especially, after attention was diverted to game theoretical models of evolution [7] and the idea of an ESS became central in evolutionary game theory. The central idea of evolution i.e. survival of the fittest is now be formulated as a mathematical algorithm usually known as replicator dynamic. We suggest recent progress in quantum game theory allows evolutionary ideas to enter and have a role in situations generally believed to lie in the domain of quantum mechanics. This combination of evolutionary ideas in quantum settings is interesting from several perspectives. Quantum considerations in the evolution of genetic code and genetic algorithms in which replicators receive their payoffs via quantum strategies are two questions [5] where evolutionary ideas can be incorporated in quantum game like situations. Another possible relevance is the competing chemical reactions in life molecules treated as players in a game. A winning chemical reaction corresponding to life hints a role of quantum mechanics because quantum strategies have been recently shown to be more effective their classical counterparts [1, 15].

Population approach borrowed from evolutionary game theory with its central idea of an ESS combined with recent developments in quantum game theory provides a new approach to certain questions relating to role of quantum mechanics in life. The analysis of the RSP game from evolutionary point of view is an example where 'stability' comes to a classical NE when players revert to quantum strategies. The 'stability' is with respect to an invasion by mutants appearing in small numbers. This stability of NE coming out of quantization can have a relevance in all the three situations indicated above. 


\section{Conclusion}

We explored evolutionary stability in a modified Rock-Scissors-Paper quantum game. We showed that a mixed strategy NE not an ESS in classical version of the game can be made an ESS when the two player play instead a quantum game by using a selected form of initial entangled state on which they apply unitary operators in their possession. Quantum mechanics, thus, gives stability to a classical mixed NE against invasion by mutants. Stability against mutants for a mixed classical NE can be made to disappear in certain types of three player symmetric games when players decide to resort to quantum strategies [5]. Stability against mutants in pair-wise contests coming as a result of quantum strategies have been shown a possibility for only pure strategies in certain type of symmetric games [3]. Our results imply the selected method of quantization [1] can bring stability against mutants to a classical mixed NE in pair-wise symmetric contests when the classically available number of pure strategies to

a player is increased to three from two. A different behavior is also observed of mixed NE from pure NE in relation to quantization.

\section{References}

[1] L. Marinatto and T. Weber, Phys. Lett. A 272, 291 (2000)

[2] K. Prestwich. Game Theory. Department of Biology, College of the Holy Cross, Worcester, MA, USA 01610. 1999.

[3] A. Iqbal and A.H. Toor, Phys. Lett. A 280/5-6, 249 (2001)

[4] A. Iqbal and A.H. Toor, Phys Lett. A 286/4, 245 (2001)

[5] A. Iqbal and A.H. Toor, preprint quant-ph/0103085

[6] Maynard Smith, J. and Price, G.R. (1973). The Logic of Animal Conflict. Nature, 246, 15-18

[7] J.W. Weibull, Evolutionary game theory. The MIT Press, Cambridge, 1995

[8] L. Marinatto, Personal correspondence.

[9] S.C. Benjamin, preprint quant-ph/0008127

[10] J. Oechssler and F. Riedel. On the dynamic foundations of evolutionary stability in continuous models. Discussion paper 7/2000. Bonn Graduate School of Economics, University of Bonn. Adenauerallee 24-42, D-53113 Bonn.

[11] Fröhlich H. (1983) Coherent Excitations in Biological Systems, SpringerVerlag, Berlin.

[12] Farhi E. \& Gutmann, S. Phys. Rev. A58, 915 (1998). 
[13] Bashford, J.D., Jarvis, P.D. \& Tsohantjis, I. (1998) Supersymmetry in the genetic code, in Physical Applications and Mathematical Aspects of Geometry, eds. H.-D. Doebner, P. Nattermann, W. Scherer and C. Schulte, World Scientific Press, Singapore.

[14] Apoorva Patel. Pramana 56 (2001) 367-381

[15] J. Eisert, M. Wilkens, M. Lewenstein, Phys. Rev. Lett. 83, 3077 (1999). Also J. Eisert and M. Wilkens, J. Mod. Opt. 47, 2543 (2000)

[16] D.A. Meyer, Phys. Rev. Lett. 82, 1052 (1999)

[17] R.F.Werner, Phy. Rev, A 58, 1827 (1998)

[18] R. Dawkins. The Selfish Gene. Oxford University Press, Oxford, 1976 